\title{
The Parameterized Complexity of Local Consistency*
}

\author{
Serge Gaspers and Stefan Szeider \\ Institute of Information Systems \\ Vienna University of Technology, Vienna, Austria \\ gaspers@kr.tuwien.ac.at, stefan@szeider.net
}

\begin{abstract}
We investigate the parameterized complexity of deciding whether a constraint network is $k$ consistent. We show that, parameterized by $k$, the problem is complete for the complexity class co-W[2]. As secondary parameters we consider the maximum domain size $d$ and the maximum number $\ell$ of constraints in which a variable occurs. We show that parameterized by $k+d$, the problem drops down one complexity level and becomes co-W[1]-complete. Parameterized by $k+d+\ell$ the problem drops down one more level and becomes fixed-parameter tractable. We further show that the same complexity classification applies to strong $k$-consistency, directional $k$-consistency, and strong directional $k$-consistency.

Our results establish a super-polynomial separation between input size and time complexity. Thus we strengthen the known lower bounds on time complexity of $k$-consistency that are based on input size.
\end{abstract}

\section{Introduction}

Local consistency is one of the oldest and most fundamental concepts of constraint solving and can be traced back to Montanari's 1974 paper [24]. If a constraint network is locally consistent, then consistent instantiations to a small number of variables can be consistently extended to an additional variable. Hence local consistency avoids certain dead-ends in the search tree, in some cases it even guarantees backtrack-free search $[1,20]$. The simplest and most widely used form of local consistency is arc-consistency, introduced by Mackworth [23], and later generalized to $k$-consistency by Freuder [19]. A constraint network is $k$-consistent if each consistent assignment to $k-1$ variables can be consistently extended to any additional $k$-th variable.

Consider a constraint network of input size $s$ where the constraints are given as relations. It is easy to see that $k$-consistency can be checked by brute force in time $O\left(s^{k}\right)$ [10]. Hence, if $k$ is a fixed constant, the check is polynomial. However, the algorithm runs in "nonuniform" polynomial time in the sense that the order of the polynomial depends on $k$, hence the running time scales poorly in $k$ and becomes impractical already for $k \geq 3$. Also more sophisticated algorithms for $k$-consistency achieve only a nonuniform polynomial running time [8].

In this paper we investigate the possibility of a uniform polynomial-time algorithm for $k$-consistency, i.e., an algorithm of running time $O\left(f(k) s^{c}\right)$ where $f$ is an arbitrary function and $c$ is a constant independent of $k$. We carry out our investigations in the theoretical framework of parameterized complexity $[15,17,25]$ which allows to distinguish between uniform and nonuniform polynomial time. Problems that can be solved in uniform polynomial time are called fixed-parameter tractable (FPT), problems that can be solved in nonuniform polynomial time are further classified within a hierarchy of parameterized complexity classes forming the chain FPT $\subseteq \mathrm{W}[1] \subseteq \mathrm{W}[2] \subseteq \mathrm{W}[3] \subseteq \cdots$, where all inclusions are believed to be strict.

\footnotetext{
${ }^{*}$ This research was funded by the ERC (COMPLEX REASON, 239962).
} 
Results We pinpoint the exact complexity of $k$-consistency decision in general and under restrictions on the given constraint network in terms of domain size $d$ and the maximum number $\ell$ of constraints in which a variable occurs.

We show that deciding $k$-consistency is co-W[2]-complete for parameter $k$, co-W[1]-complete for parameter $k+d$, and fixed-parameter tractable for parameter $k+d+\ell$. Hence, subject to complexity theoretic assumptions, $k$-consistency cannot be decided in uniform polynomial-time in general, but admits a uniform polynomial-time solution if domain size and variable occurrence are bounded. The hardness results imply a super-polynomial separation between input size and running time for $k$ consistency algorithms.

We further show that all three complexity results also hold for deciding strong $k$-consistency, for deciding directional $k$-consistency, and for deciding strong directional $k$-consistency. A constraint network is strongly $k$-consistent if it is $j$-consistent for all $1 \leq j \leq k$. Directional local consistency takes a fixed ordering of the variables into account, the variable to which a local instantiation is extended is ordered higher than the previously instantiated variables [12].

Known Lower Bounds In previous research, lower bounds on the running time of $k$-consistency algorithms have been obtained $[8,10]$. These lower bounds are based on instances of large input size, and the observation that any $k$-consistency algorithm needs to read the entire input. For instance, to decide whether a given constraint network on $n$ variables is $k$-consistent one needs to check each constraint of arity $r \leq k$ at least once (the arity of a constraint is the number of variables that occur in the constraint). Since there can be $\sum_{i=1}^{k}\left(\begin{array}{c}n \\ i\end{array}\right)$ such constraints, $\Omega\left(n^{k}\right)$ provides a lower bound on the running time of any $k$-consistency algorithm. Taking the domain size $d$ into account, this lower bound can be improved to $\Omega\left((d n)^{k}\right)$ [10]. However, the constraint networks to which this lower bound applies are of size $s=\Omega\left((d n)^{k}\right)$. Therefore the known lower bounds do not provide a separation between input size and running time.

\section{Preliminaries}

\subsection{Constraint Networks and Local Consistency Problems}

A constraint network (or CSP instance) $N$ is a triple $(X, D, C$ ), where $X$ is a finite set of variables, $D$ is a finite set of values, and $C$ is a finite set of constraints. Each constraint $c \in C$ is a pair $(S, R)$, where $S=\operatorname{var}(C)$, the constraint scope, is a finite sequence of distinct variables from $X$, and $R$, the constraint relation, is a relation over $D$ whose arity matches the length of $S$, i.e., $R \subseteq D^{r}$ where $r$ is the length of $S$. The size of $N$ is $s=|N|=|X|+|D|+\sum_{(S, R) \in C}|S| \cdot(1+|R|)$.

Let $N=(X, D, C)$ be a constraint network. A partial instantiation of $N$ is a mapping $\alpha: X^{\prime} \rightarrow D$ defined on some subset $\operatorname{var}(\alpha)=X^{\prime} \subseteq X$. We say that $\alpha$ satisfies a constraint $c=\left(\left(x_{1}, \ldots, x_{r}\right), R\right) \in$ $C$ if $\operatorname{var}(c) \subseteq \operatorname{var}(\alpha)$ and $\left(\alpha\left(x_{1}\right), \ldots, \alpha\left(x_{r}\right)\right) \in R$. If $\alpha$ satisfies all constraints of $N$ then it is a solution of $N$; in this case, $N$ is satisfiable. We say that $\alpha$ is consistent with a constraint $c \in C$ if either $\operatorname{var}(c)$ is not a subset of $\operatorname{var}(\alpha)$ or $\alpha$ satisfies $c$. If $\alpha$ is consistent with all constraints of $N$ we call it consistent. The restriction of a partial assignment $\alpha$ to a set of variables $Y$ is denoted $\left.\alpha\right|_{Y}$. It has scope $\operatorname{var}(\alpha) \cap Y$ and $\left.\alpha\right|_{Y}(x)=\alpha(x)$ for all $x \in \operatorname{var}\left(\left.\alpha\right|_{Y}\right)$.

Let $k>0$ be an integer. A constraint network $N=(X, D, C)$ is $k$-consistent if for all consistent partial instantiations $\alpha$ of $N$ with $|\operatorname{var}(\alpha)|=k-1$ and all variables $x \in X \backslash \operatorname{var}(\alpha)$ there is a consistent partial instantiation $\alpha^{\prime}$ such that $\operatorname{var}\left(\alpha^{\prime}\right)=\operatorname{var}(\alpha) \cup\{x\}$, and $\left.\alpha^{\prime}\right|_{\operatorname{var}(\alpha)}=\alpha$. In such a case we say that $\alpha^{\prime}$ consistently extends $\alpha$ to $x$. A constraint network is strongly $k$-consistent if it is $j$-consistent for all $j=1, \ldots, k$.

For further background on local consistency we refer to other sources $[2,11]$.

We consider the following decision problem.

$k$-CONSISTENCY

Input: $\quad$ A constraint network $N=(X, D, C)$ and an integer $k>0$.

Question: Is $N k$-consistent? 
The problem Strong $k$-CONSISTENCY is defined analogously, asking whether $N$ is strongly $k$-consistent.

It is easy to see that $k$-CONSISTENCY is co-NP-hard if $k$ is unbounded. Take an arbitrary constraint network $N=(X, D, C)$ and form a new network $N^{\prime}$ from $N$ by adding a new variable $x$, and $|X|+1$ new constraints with empty relations, namely the constraint whose scope contains all variables, and all possible constraints of arity $|X|$ having $x$ in their scope. Let $k=|X|+1$. Now $N^{\prime}$ is $k$-consistent if and only if $N$ is not satisfiable. Since $k$ is large this reduction seems somehow unnatural and breaks down for bounded $k$. This suggests to "deconstruct" this hardness proof (in the sense of [22]) and to parameterize by $k$.

The constraint network $N$ is directionally $k$-consistent with respect to a total order $\leq$ on its variables if every consistent partial instantiation $\alpha$ of $k-1$ variables of $N$ can be consistently extended to every variable that is higher in the order $\leq$ than any variable of $\operatorname{var}(\alpha)$. The corresponding decision problem is defined as follows.

\section{DiRECTIONAL $k$-CONSISTENCY}

Input: $\quad$ A constraint network $N=(X, D, C)$, a total order $\leq$ on $X$, and an integer $k>0$.

Question: Is $N$ directionally $k$-consistent with respect to $\leq$ ?

A constraint network is strongly directionally $k$-consistent if and only if it is directionally $j$-consistent for all $j=1, \ldots, k$. The strong counterpart of the DIRECTIONAL $k$-CONSISTENCY problem is called Strong Directional $k$-Consistency.

We will consider parameterizations of these four problems by $k$, by $k+d$, and by $k+d+\ell$, where $d=|D|$ and $\ell$ denotes the maximum number of constraints in which a variable occurs.

\subsection{Parameterized Complexity}

We define the basic notions of Parameterized Complexity and refer to other sources $[15,17]$ for an in-depth treatment. A parameterized problem can be considered as a set of pairs $(I, k)$, the instances, where $I$ is the main part and $k$ is the parameter. The parameter is usually a non-negative integer. A parameterized problem is fixed-parameter tractable if there exists an algorithm that solves any instance $(I, k)$ of size $n$ in time $f(k) n^{O(1)}$, where $f$ is a computable function. FPT denotes the class of all fixed-parameter tractable decision problems.

Parameterized complexity offers a completeness theory, similar to the theory of NP-completeness, that allows the accumulation of strong theoretical evidence that some parameterized problems are not fixed-parameter tractable. This theory is based on a hierarchy of complexity classes

$$
\mathrm{FPT} \subseteq \mathrm{W}[1] \subseteq \mathrm{W}[2] \subseteq \mathrm{W}[3] \subseteq \cdots .
$$

where all inclusions are believed to be strict. Each class $\mathrm{W}[i]$ contains all parameterized decision problems that can be reduced to a canonical parameterized satisfiability problem $P_{i}$ under parameterized reductions. These are many-to-one reductions where the parameter for one problem maps into the parameter for the other. More specifically, a parameterized problem $L$ reduces to a parameterized problem $L^{\prime}$ if there is a mapping $R$ from instances of $L$ to instances of $L^{\prime}$ such that

1. $(I, k)$ is a Yes-instance of $L$ if and only if $\left(I^{\prime}, k^{\prime}\right)=R(I, k)$ is a Yes-instance of $L^{\prime}$,

2. there is a computable function $g$ such that $k^{\prime} \leq g(k)$, and

3. there is a computable function $f$ and a constant $c$ such that $R$ can be computed in time $O(f(k)$. $\left.n^{c}\right)$, where $n$ denotes the size of $(I, k)$.

A parameterized problem $L$ is then in $\mathrm{W}[i], i \in \mathbb{N}$, if it has a parameterized reduction to the problem of deciding whether a Boolean decision circuit (a decision circuit is a circuit with exactly one output), with AND, OR, and NOT gates, of constant depth such that on each path from an input to the output, all but $i$ gates have a constant number of inputs, parameterized by the number of ones in a satisfying assignment to the inputs of the circuit [15]. 
A parameterized problem is in co- $\mathrm{W}[i], i \in \mathbb{N}$, if its complement is in $\mathrm{W}[i]$, where the complement of a parameterized problem is the parameterized problem resulting from reversing the YES and No answers.

If any co- $\mathrm{W}[i]$-complete problem is fixed-parameter tractable, then co- $\mathrm{W}[i]=\mathrm{FPT}=\mathrm{co}-\mathrm{FPT}=$ $\mathrm{W}[i]$ follows, which causes the Exponential Time Hypothesis to fail [17]. Hence co-W[i]-completeness provides strong theoretical evidence that a problem is not fixed-parameter tractable.

\subsection{Tries, Turing Machines, and Gaifman Graphs}

Tries A trie $[9,18]$ is a tree for storing strings in which there is one node for every prefix of a string. Let $T$ be a trie that stores a set $S$ of strings on an alphabet $\Sigma$. At a given node $v$ of $T$, corresponding to the prefix $p(v)$, there is an array with one entry for every character $c$ of $\Sigma$. If $p(v) . c$ is a prefix of a string of $S$, the entry corresponding to $c$ has a pointer to the node corresponding to the prefix $p(v) . c$ (the dot denotes a concatenation). If $p(v) . c$ is not a prefix of a string of $S$, the entry corresponding to $c$ has a null pointer. Thus, a trie uses space $O(|S| \cdot|\Sigma|)$, while inserting or searching a string $s$ can be done in time $O(|s|)$ using the ordinal values for characters as array indices.

Turing Machines A nondeterministic Turing Machine $(N T M)[4,17]$ with $t$ tapes is an 8-tuple $M=\left(Q, \Gamma, \beta, \$, \Sigma, \delta, q_{0}, F\right)$, where

- $Q$ is a finite set of states,

- the tape alphabet $\Gamma$ is a finite set of symbols,

- $\beta \in \Gamma$ is the blank symbol, the only symbol allowed to occur on the tape(s) infinitely often,

- $\$ \in \Gamma$ is a delimiter marking the (left) end of a tape,

- $\Sigma \subseteq \Gamma$ is the set of input symbols,

- $q_{0} \in Q$ is the initial state,

- $F \subseteq Q$ is the set of final states,

- $\sigma \subseteq Q \backslash F \times \Gamma^{t} \times Q \times \Gamma^{t} \times\{L, N, R\}^{t}$ is the transition relation. A transition $\left(q,\left(a_{1}, \ldots, a_{t}\right), q^{\prime},\left(a_{1}^{\prime}, \ldots, a_{t}^{\prime}\right),\left(d_{1}, \ldots, d_{t}\right)\right) \in \sigma$ allows the machine, when it is in state $q$ and the head of each tape $T_{i}$ is positioned on a cell containing the symbol $a_{i}$, to transition in one computation step into the state $q^{\prime}$, writing the symbol $a_{i}^{\prime}$ into the cell on which the head of each tape $T_{i}$ is positioned, and shifting this head one position to the left if $d_{i}=L$, one position to the right if $d_{i}=R$, or not at all if $d_{i}=N$. On each tape, $\$$ cannot be overwritten and allows only right transitions, which is formally achieved by imposing that whenever $\left(q,\left(a_{1}, \ldots, a_{t}\right), q^{\prime},\left(a_{1}^{\prime}, \ldots, a_{t}^{\prime}\right),\left(d_{1}, \ldots, d_{t}\right)\right) \in \sigma$, then for all $i \in\{1, \ldots, t\}$ we have $a_{i}=\$$ if and only if $a_{i}^{\prime}=\$$, and $a_{i}=\$$ implies $d_{i}=R$.

Initially, the first tape contains $\$ w \beta \beta \ldots$, where $w \in \Sigma^{*}$ is the input word, all other tapes contain $\$ \beta \beta \ldots, M$ is in state $q_{0}$, and all heads are positioned on the first cell to the right of the $\$$ symbol. We speak of a single-tape NTM if $t=1$ and of a multi-tape NTM if $t>1$. $M$ accepts the input word $w$ in $k$ steps if there exists a transition path that takes $M$ with input word $w$ to a final state in $k$ computation steps.

Graphs The Gaifman graph $\mathcal{G}(N)$ of a constraint network $N=(X, D, C)$ has the vertex set $V(\mathcal{G}(N)):=X$ and its edge set $E(\mathcal{G}(N))$ contains an edge $\{u, v\}$ if $u$ and $v$ occur together in the scope of a constraint of $C$. In a graph $G=(V, E)$, the (open) neighborhood of a vertex $v$ is the subset of vertices sharing an edge with $v$ and is denoted $\Gamma(v)$, its closed neighborhood is $\Gamma[v]:=\Gamma(v) \cup\{v\}$, and the degree of $v$ is $d(v):=|\Gamma(v)|$. The maximum vertex degree of $G$ is denoted $\Delta(G)$. For a vertex set $S, \Gamma[S]:=\bigcup_{v \in S} \Gamma[v] . S$ is independent in $G$ if no two vertices of $S$ are adjacent in $G$. $S$ is dominating in $G$ if $\Gamma[S]=V$. 


\section{$3 \quad k$-Consistency Parameterized by $k$}

In this section, we consider the most natural parameterization of $k$-ConsISTENCY. Theorem 1 shows that the problem is co-W[2]-hard, parameterized by $k$, and Theorem 2 shows that it is in co-W[2]. These results are also extended to the strong and directional versions of the problem, resulting in Corollary 1, which says that all four problems are co-W[2]-complete when parameterized by $k$.

Theorem 1. Parameterized by $k$, the following problems are co-W[2]-hard: $k$-Consistency, Strong $k$-Consistency, Directional $k$-Consistency, and Strong Directional $k$-Consistency.

Proof. We show a parameterized reduction from Independent Dominating Set to the complement of $k$-Consistency. The Independent Dominating Set problem was shown to be W[2]-hard by Downey and Fellows [13] (see also [7] where W[2]-completeness is established).

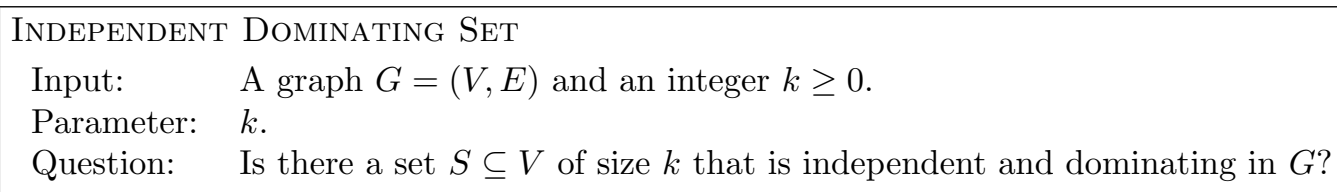

Let $G=(V, E)$ and $k \geq 0$ be an instance of Independent Dominating Set. We construct a constraint network $N=(X, D, C)$ as follows. We take $k+1$ variables and put $X=\left\{x_{1}, \ldots, x_{k+1}\right\}$. For $1 \leq i \leq k+1$ we put $D\left(x_{i}\right)=V$. The set $C$ contains $\left(\begin{array}{c}k+1 \\ 2\end{array}\right)$ constraints $c_{i, j}=\left(\left(x_{i}, x_{j}\right), R_{E}\right)$, $1 \leq i<j \leq k+1$, where $R_{E}=\{(v, u) \in V \times V \mid u \neq v,\{u, v\} \notin E\}$. This completes the definition of the constraint network $N$.

Claim 1. $G$ has an independent dominating set of size $k$ if and only if $N$ is not $(k+1)$-consistent.

To show the $(\Rightarrow)$-direction, suppose $S=\left\{v_{1}, \ldots, v_{k}\right\}$ is an independent dominating set of $G$. Consider the partial instantiation $\alpha$ with $\operatorname{var}(\alpha)=\left\{x_{1}, \ldots, x_{k}\right\}$ and $\alpha\left(x_{i}\right)=v_{i}, 1 \leq i \leq k$.

First we show that $\alpha$ is consistent. Consider an arbitrary constraint $c_{i, j}$ with $\operatorname{var}\left(c_{i, j}\right) \subseteq \operatorname{var}(\alpha)$. It follows that $1 \leq i<j \leq k$. Since $S$ is an independent set, $\left\{v_{i}, v_{j}\right\} \notin E$, and so $\left(\alpha\left(x_{i}\right), \alpha\left(x_{j}\right)\right)=$ $\left(v_{i}, v_{j}\right) \in R_{E}$. Hence $\alpha$ is consistent.

Second, we show that $\alpha$ cannot be consistently extended to $x_{k+1}$. Let $\alpha^{\prime}$ be an arbitrarily chosen partial instantiation of $N$ with $\operatorname{var}\left(\alpha^{\prime}\right)=\left\{x_{1}, \ldots, x_{k+1}\right\}$ extending $\alpha$. Let $v_{k+1}=\alpha^{\prime}\left(x_{k+1}\right)$. Since $S$ is a dominating set of $G$, there must be some $1 \leq i \leq k$ with $\left\{v_{i}, v_{k+1}\right\} \in E$. Consequently $\left(\alpha^{\prime}\left(x_{i}\right), \alpha^{\prime}\left(x_{k+1}\right)\right)=\left(v_{i}, v_{k+1}\right) \notin R_{E}$, hence $\alpha^{\prime}$ is not consistent with $c_{i, k+1}$. Since $\alpha^{\prime}$ was chosen arbitrarily, we conclude that $\alpha$ cannot be consistently extended to $x_{k+1}$. Hence $N$ is not $(k+1)$ consistent.

It remains to show the $(\Leftarrow)$-direction. Assume that $N$ is not $(k+1)$-consistent. Hence there is a partial instantiation $\alpha$ on $k$ variables that cannot be consistently extended to a further variable $x$. Without loss of generality, assume $\operatorname{var}(\alpha)=\left\{x_{1}, \ldots, x_{k}\right\}$ and $x=x_{k+1}$. Let $v_{i}=\alpha\left(x_{i}\right), 1 \leq i \leq k$. Since $\alpha$ is consistent, it follows that $S=\left\{v_{1}, \ldots, v_{k}\right\}$ is an independent set of $G$. Furthermore, $v_{i} \neq v_{j}$ for $1 \leq i<j \leq k$, since $R_{E}$ does not contain any pair of the form $(v, v)$. Hence $|S|=k$. It remains to show that $S$ is dominating. Assume to the contrary that some $v_{k+1} \in V \backslash S$ is not dominated by $S$, i.e., $v_{k+1}$ is not a neighbor of any vertex in $S$. This however, implies that the extension $\alpha^{\prime}$ of $\alpha$ with $\alpha\left(x_{k+1}\right)=v_{k+1}$ is consistent, contradicting our assumption. Hence $S$ is indeed an independent dominating set of size $k$, and Claim 1 is shown true.

Evidently $N$ can be obtained from $G$ in polynomial time. Thus we have established a parameterized reduction from Independent Dominating Set to the complement of $k$-Consistency. The co-W[2]hardness of $k$-CONSISTENCY, parameterized by $k$, now follows from the W[2]-hardness of InDEPENDent Dominating Set.

The co-W[2]-hardness of Strong $k$-Consistency, parameterized by $k$, is proved analogously by reducing from the variant of Independent Dominating Set which asks for an independent dominating set of size at most $k$. This variant is also W[2]-hard, as shown by Downey et al. [16]. 
To show that the directional versions of the problem are co-W[2]-hard, parameterized by $k$, we use the same reductions and additionally specify a total ordering of the vertices. We use the total order by increasing indices of the variables, and observe that the variable to which the partial order $\alpha$ cannot be extended is the last variable in this order in both directions of the proof of Claim 1. Thus, this modification of the reductions shows that Directional $k$-Consistency and Strong Directional $k$-CONSISTENCY are also co-W[2]-hard parameterized by $k$.

The reductions of Theorem 1 actually show somewhat stronger results, namely that the four problems are co-W[2]-hard when parameterized by $k+\ell$. This follows from the observation that the number of variables in the target problems is $k+1$. From Theorem 2, the co-W[2]-membership of this parameterization will follow. Thus, the problems are co-W[2]-complete when parameterized by $k+\ell$.

For the co-W[2]-membership proof, we build a multi-tape nondeterministic Turing machine that reaches a final state in $f(k)$ steps, for some function $f$, if and only if $N$ is not $k$-consistent. As this reduction needs to be a parameterized reduction, we need avoid that the size of the Turing machine (and the time needed to compute it) depends on $O\left(|X|^{k}\right)$ or $O\left(d^{k}\right)$ terms, which would have been very handy to model constraint scopes and constraint relations. We counter this issue via organizing the states of the NTM in tries. There is a first level of tries to determine whether a certain subset of variables is the scope of some constraint. There is a second level of tries to find out whether a certain partial instantiation is allowed by a constraint relation. A second issue that needs particular attention is the size of the transition table. The number of tapes of the NTM is $d+4$, and we cannot afford a transition for each combination of characters that the head of each tape might be positioned on. We use Cesati's information hiding trick [4] to avoid this issue, which means that the machine does the computations in such a way that in each state, it knows for most tapes (i.e., all, except a constant number of tapes) which characters are in the cell on which the corresponding head is positioned.

Theorem 2. Parameterized by $k$, the following problems are in co-W[2]: $k$-Consistency, Strong $k$-Consistency, Directional $k$-Consistency, and Strong Directional $k$-Consistency.

Proof. Cesati [4] showed that the following parameterized problem is in W[2].

\begin{tabular}{ll}
\hline ShorT Multi-TAPE NTM COMPUTATION \\
Input: & A multi-tape NTM $M$, a word $w$ on the input alphabet of $M$, and an integer \\
& $k>0$. \\
Parameter: & $k$. \\
Question: & Does $M$ accept $w$ in at most $k$ steps?
\end{tabular}

We reduce the complement of $k$-Consistency to Short Multi-tape NTM Computation. Let $(N=(X, D, C), k)$ be an instance for $k$-ConsistenCY. We will construct an instance $\left(M, w, k^{\prime}\right)$ which is a Yes-instance for Short Multi-Tape NTM Computation if and only if $(N, k)$ is a No-instance for $k$-CONSISTENCY.

Let us describe how $M=\left(Q, \Gamma, \beta, \$, \Sigma, q_{0}, F, \sigma\right)$ operates. $M$ has $d+4$ tapes, named $G x, G d, G x_{k}, S, d_{1}, \ldots, d_{d}$, and the input word $w$ is empty. Thus, all the information about $N$ is encoded in the states and transitions of $M$. The tape alphabet of $M$ is $\Gamma=\{\beta, \$\} \cup X \cup D \cup\{T, F, 1,0\}$.

In the initialization phase, $M$ writes a ' $T$ ' symbol on the tapes $d_{1}, \ldots, d_{d}$ and it positions the head of each tape on the first blank symbol of this tape. This can be done in one computation step.

In the guess phase, $M$ nondeterministically guesses $x(1), \ldots, x(k) \in X$ such that $x(i)<x(i+1)$ for all $i \in\{1, \ldots, k-2\}$, and it guesses $d(1), \ldots, d(k-1) \in D$. Here, $\leq$ is an arbitrary order on the variables, and $a<b$ means $a \leq b$ and $a \neq b$. It appends $x(1), \ldots, x(k-1)$ to the tape $G x$, it appends $d(1), \ldots, d(k-1)$ to the tape $G d$, and it appends $x(k)$ to the tape $G x_{k}$. The goal is to make $M$ halt in a final state after a number of steps only depending on $k$ if and only if the partial instantiation $\alpha$, with $\alpha(x(i))=d(i), 1 \leq i \leq k-1$, is consistent, but $\alpha$ cannot be consistently extended to $x(k)$. See Figure 1 for a typical content of the tapes during the execution of $M$.

The remaining states of $M$ are partitioned into $|X|$ parts, one part for each choice of $x(k) . M$ reads $x(k)$ on the tape $G x_{k}$ and moves to the initial state in the part corresponding to $x(k)$. 


$\begin{array}{rllllll}G x: & \$ & x(1) & x(2) & x(3) & \ldots & x(k-1) \\ G d: & \$ & d(1) & d(2) & d(3) & \ldots & d(k-1) \\ G x_{k}: & \$ & x(k) & & & & \\ S: & \$ & 0 & 0 & 1 & \ldots & 0 \\ d_{1}: & \$ & T & F & F & & \\ d_{2}: & \$ & T & & & & \\ d_{3}: & \$ & T & F & & & \\ \ldots & & & & & & \\ d_{d}: & \$ & T & F & F & \end{array}$

Figure 1: A typical content of the tapes during an execution of $M$ (blank symbols are omitted).

On the $S$ tape, $M$ now enumerates all binary 0/1 strings of length $k-1$. The strings in $\{0,1\}^{k-1}$ represent subsets of $\{x(1), \ldots, x(k-1)\}$, i.e., all possible scopes of the constraints that could be violated by the partial instantiation $\alpha$. For each such binary string, representing a subset $X^{\prime}$ of $\{x(1), \ldots, x(k-1)\}, M$ moves to a state representing $X^{\prime}$ if there is a constraint with scope $X^{\prime}$, otherwise it moves to a state calculating the next subset $X^{\prime}$. This is achieved by a trie of states; each node of this trie represents a subset $X^{\prime \prime}$ of $X$ which is the subset of the first few variables of the scope of some constraint (i.e., $X^{\prime \prime}$ represents the prefix of a constraint scope, if we imagine all constraint scopes to be strings of increasing variable names). Thus, the size of this trie does not exceed $O(|C| \cdot|X|)$, and the node corresponding to $X^{\prime}$ (or the evidence that there is no node corresponding to $\left.X^{\prime}\right)$ is found in $O\left(\left|X^{\prime}\right|\right)=O(k)$ steps. Without loss of generality, we may assume that for each subset of $X$, there is at most one constraint with that scope; otherwise merge constraints with the same scope. If there is a node representing $X^{\prime}$, there is a constraint $c$ with scope $X^{\prime}$. A trie of states starting at this node represents all tuples that belong to the constraint relation $R$ of $c$. This trie has size $O\left(|R| \cdot\left|X^{\prime}\right|\right)$. Moreover, $M$ can determine in time $O\left(\left|X^{\prime}\right|\right)$ whether the tuple $t$, setting $x(i)$ to $d(i)$ for each $i$ such that $x(i) \in X^{\prime}$, is in $R$. If so, it moves to a state representing $t$, otherwise it moves to a non-accepting state where is loops forever (as the selected partial instantiation $\alpha$ is not consistent). At the state representing $t$, it appends ' $F$ ' to all tapes $d_{j}$ such that there exists a constraint with scope $X^{\prime} \cup\{x(k)\}$ and its constraint relation does not contain the tuple setting $x(i)$ to $d(i)$ for each $x(i) \in X^{\prime}$ and setting $x(k)$ to $d_{j}$. Then, it moves to the state computing the next set $X^{\prime}$. The machine can only move to a final state if the last symbol on each $d_{i}$-tape is ' $F$ ', meaning that the calculated partial instantiation $\alpha(x(i))=d(i), 1 \leq i \leq k-1$ is consistent (otherwise the machine loops forever in a non-accepting state), but cannot be consistently extended to $x(k)$ (otherwise some $d_{i}$-tape does not end in ' $F$ '), which certifies that $(N, k)$ is a No-instance for $k$-ConsistenCY.

The number of states of $M$ is clearly polynomial in $|N|+k$. The transition relation has also polynomial size as we use Cesati's information hiding trick [4], and place the head of the tapes $d_{1}, \ldots, d_{d}$ always on the first blank symbol, except for the final check of whether $M$ moves into a final state. If the machine can reach a final state, it can reach one in a number of steps which is a function of $k$ only. This proves the co-W[2]-membership of $k$-CONSISTENCY, parameterized by $k$.

Checking whether a network is a No-instance for STRONG $k$-CONSISTENCY can be done by checking whether it is a No-instance for $j$-Consistency for some $j \in\{1, \ldots, k\}$. Thus, it is sufficient to build $k$ NTMs as we described, one for each value of $j \in\{1, \ldots, k\}$, nondeterministically guess the integer $j$ for which $N$ is not $j$-consistent in case $N$ is a No-instance, and move to the initial state of the $j^{\text {th }}$ NTM checking whether $N$ is a No-instance for $j$-Consistency. Thus, STrong $k$-Consistency parameterized by $k$ is in co-W[2].

For the directional variants of the problem, the order $\leq$ is the one given in the input. It is sufficient to additionally require $x(k)$ to represent a variable that is higher in the order $\leq$ than all variables $x(1), \ldots, x(k-1)$. Thus, our condition that $x(i)<x(i+1)$ for all $i \in\{1, \ldots, k-2\}$ is extended to $i \in\{1, \ldots, k-1\}$. We conclude that the parameterizations of DiRECTIONAL $k$-Consistency and Strong Directional $k$-Consistency by $k$ are in co-W[2] as well. 
From Theorems 1 and 2, we obtain the following corollary.

Corollary 1. Parameterized by $k$, the following problems are co-W[2]-complete: $k$-Consistency, Strong $k$-Consistency, Directional $k$-Consistency, and Strong Directional $k$-ConsisTENCY.

As mentioned before, the corollary also holds for the parameterization by $k+\ell$.

\section{$4 k$-Consistency Parameterized by $k+d$}

In our quest to find parameterizations that make local consistency problems tractable, we augment the parameter by the domain size $d$. We find that, with this parameterization, the problems become co-W[1]-complete. The co-W[1]-hardness follows from a parameterized reduction from INDEPENDENT SET.

Theorem 3. Parameterized by $k+d$, the following problems are hard for co-W[1]: $k$-Consistency, Strong $k$-Consistency, Directional $k$-Consistency, and Strong Directional $k$-ConsisTENCY.

Proof. To show that the complement of $k$-Consistency is W[1]-hard, we reduce from IndePendent SET, which is well-known to be W[1]-hard [14].

\begin{tabular}{|ll|}
\hline INDEPENDENT & SET \\
Input: & A graph $G=(V, E)$ and an integer $k \geq 0$. \\
Parameter: & $k$. \\
Question: & Is there an independent set of size $k$ in $G ?$ \\
\hline
\end{tabular}

Let $G=(V, E)$ with $V=\left\{v_{1}, \ldots, v_{n}\right\}$ and $k \geq 0$ be an instance of Independent Set. We construct a constraint network $N=(X, D, C)$ as follows.

The set of variables is $X=\left\{x_{1}, \ldots, x_{n}, c\right\}$. The set of values is $D=\{0, \ldots, k\}$. The constraint set $C$ contains the constraints

(a) $\left(\left(x_{i}, x_{j}\right),\{(a, b): a, b \in\{0, \ldots, k\}\right.$ and $(a=0$ or $\left.b=0)\}\right)$, for all $v_{i} v_{j} \in E$, constraining at least one of $x_{i}$ and $x_{j}$ to take the value 0 if $v_{i} v_{j} \in E$,

(b) $\left(\left(x_{i}, c\right),\{(a, b): a, b \in\{0, \ldots, k\}\right.$ and $(a=0$ or $\left.a \neq b)\}\right)$, for all $i \in\{1, \ldots, n\}$, constraining $c$ to be set to a value different from $j$ if any $x_{i}$ is set to $j>0$, and

(c) $((c),\{(1), \ldots,(k)\})$, restricting the domain of $c$ to $\{1, \ldots, k\}$.

This completes the definition of the constraint network $N$. See Figure 2 for an illustration of $N$.

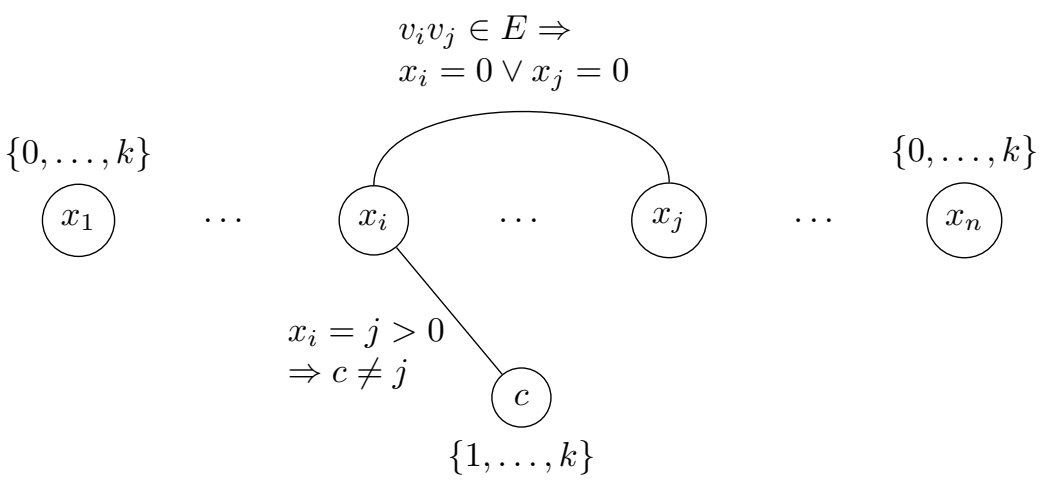

Figure 2: The target constraint network in the parameterized reduction from IndEPENDENT SET. 
Claim 2. $G$ has an independent set of size $k$ if and only if $N$ is not $(k+1)$-consistent.

To show the $(\Rightarrow)$-direction, suppose $S=\left\{v_{s(1)}, \ldots, v_{s(k)}\right\}$ is an independent set in $G$. Consider the partial instantiation $\alpha$ such that $\alpha\left(x_{s(i)}\right)=i, i=1, \ldots, k$. This partial instantiation is consistent, but cannot be consistently extended to $c$.

To show the $(\Leftarrow)$-direction, suppose $\alpha$ is a consistent partial instantiation of $k$ variables and $x$ is a variable such that $\alpha$ cannot be consistently extended to $x$. As the only constraint preventing a variable to be set to 0 is the constraint $(c)$ restricting the domain of $c$ to $\{1, \ldots, k\}$, we have that $x=c$. Now, that $c$ cannot take any of the values in $\{1, \ldots, k\}$ is achieved by the constraints of type (b) by having $\alpha$ bijectively map $k$ variables $x_{s(1)}, \ldots, x_{s(k)}$ to the set $\{1, \ldots, k\}$ without violating any constraint. As two distinct vertices can only be assigned values different from 0 each if they are not adjacent, by the constraints of type $(a)$, we have that $\left\{x_{s(1)}, \ldots, x_{s(k)}\right\}$ is an independent set of size $k$. Hence Claim 2 is shown true.

Evidently $N$ can be obtained from $G$ in polynomial time. Thus we have established a parameterized reduction from IndEPEndent Set to the complement of $k$-Consistency with $d=k+1$. The co-W[1]-hardness of $k$-Consistency, parameterized by $k+d$, now follows from the $\mathrm{W}[1]$-hardness of INDEPENDENT SET.

For the co-W[1]-hardness of Strong $k$-Consistency, parameterized by $k+d$, just observe that any partial instantiation of fewer than $k$ variables can be extended to any other variable. Thus, $G$ has an independent set of size $k$ if and only if $N$ is not strongly $k$-consistent, and the co-W[1]-hardness of Strong $k$-Consistency, parameterized by $k+d$, follows analogously.

For the directional versions of the problem, we use the same reduction and define the ordering in the target problem to be some ordering which has $c$ as its last element. Observing that $c$ is the variable to which the partial instantiation $\alpha$ cannot be extended in both directions of the proof of Claim 2, the co-W[1]-hardness of Directional $k$-Consistency and Strong Directional $k$-Consistency, parameterized by $k+d$, follows.

It remains to show co-W[1]-membership, which easily follows from the parameterized reduction from Theorem 2 (we designed the proof of Theorem 2 in such a way that the same parameterized reduction shows co-W[1]-membership for the parameterization by $k+d)$.

Theorem 4. Parameterized by $k+d$, the following problems are in co-W[1]: $k$-Consistency, Strong $k$-Consistency, Directional $k$-Consistency, and Strong Directional $k$-ConsisTENCY.

Proof. Cesati and Di Ianni [6] showed that the following parameterized problem is in W[1] (see also [3] where $\mathrm{W}[1]$-completeness is established for the single-tape version of the problem).

\begin{tabular}{ll}
\hline Short Bounded-TAPE NTM COMPUTATION \\
Input: & A $t$-tape NTM $M$, a word $w$ on the input alphabet of $M$, and an integer $k>0$. \\
Parameter: & $k+t$. \\
Question: & Does $M$ accept $w$ in at most $k$ steps?
\end{tabular}

Now, the proof follows from the proof of Theorem 2, which gives a parameterized reduction from the four problems to Short Multi-Tape NTM Computation where the number of tapes is bounded by $d+4$.

From Theorems 3 and 4, we obtain the following corollary.

Corollary 2. Parameterized by $k+d$, the following problems are co-W[1]-complete: $k$-ConsISTENCY, Strong $k$-Consistency, Directional $k$-Consistency, and Strong Directional $k$-ConsisTENCY. 


\section{$5 \quad k$-Consistency Parameterized by $k+d+\ell$}

We further augment the parameter by $\ell$, the maximum number of constraints in which a variable occurs. For this parameterization, we are able to show that the considered problems are fixed-parameter tractable. Bounding both $d$ and $\ell$ is a reasonable restriction, as it still admits constraint networks whose satisfiability is NP-complete. For instance, determining whether a graph with maximum degree 4 is 3-colorable is an NP-complete problem [21] that can be naturally expressed as a constraint network with $d=3$ and $\ell=4$.

For checking whether there is a partial assignment that cannot be extended to a variable $x$, our FPT algorithm uses the fact that the number of constraints involving $x$ is bounded by a function of the parameter. As constraints with a scope on more than $k$ variables are irrelevant, it follows that the number of variables whose instantiation could prevent $x$ from taking some value can also be bounded by a function of the parameter. For strong $k$-consistency, these observations are already sufficient to obtain an FPT algorithm as all instantiations of subsets of size at most $k-1$ of the relevant variables can be enumerated. For (non-strong) $k$-consistency, the algorithm tries to select some independent variables to complete the consistent partial assignment, which must be of size exactly $k-1$. If such a set of independent variables does not exist, the size of the considered constraint network is actually bounded by a function of the parameter and can be solved by a brute-force algorithm.

Theorem 5. Parameterized by $k+d+\ell$, the following problems are fixed-parameter tractable: $k$-CoNsistency, Strong $k$-Consistency, Directional $k$-Consistency, and Strong Directional $k$-CONSISTENCY.

Proof. Consider an input instance $N=(X, D, C)$ for $k$-Consistency. In a first step, discard all constraints $c$ with $|\operatorname{var}(c)|>k$, as they cannot influence whether $N$ is $k$-consistent. The algorithm goes over all $|X|$ possibilities for choosing the vertex $x$ to which a consistent partial instantiation $\alpha$ on $k-1$ variables cannot be extended. If $|X| \leq k \cdot(1+k \cdot \ell)$, then the number of constraints is at most $|X| \cdot \ell \leq k \cdot(1+k \cdot \ell) \cdot \ell$ and each constraint has size at most $k \cdot\left(1+d^{k}\right)$. It follows that

$$
|N| \leq k \cdot(1+k \cdot \ell)+d+(1+k \cdot \ell) \cdot k^{2} \cdot \ell \cdot\left(1+d^{k}\right) .
$$

Thus, $N$ is a kernel, i.e., its size is a function of the parameter, and any algorithm solving $k$-ConsisTENCY for $N$ (brute-force search or Cooper's algorithm [8]) has a running time that can be bounded by a function of the parameter only.

Therefore, suppose $|X|>k \cdot(1+k \cdot \ell)$. Let $G:=\mathcal{G}(N)$ be the Gaifman graph of $N$. The algorithm chooses a set $S$ of $k-1$ variables for the scope of $\alpha$. To do this, it goes over all $\delta=0, \ldots, k-1$, where $\delta$ represents the number of variables in $S \cap \Gamma(x)$. The number of possibilities for choosing these $\delta$ variables is at most $\left(\begin{array}{c}k \cdot \ell \\ \delta\end{array}\right)$ as $d(x) \leq k \cdot \ell$. The remaining $k-1-\delta$ variables of $S$ need to be chosen from $V \backslash \Gamma[S \cup\{x\}]$. Note that these variables do not influence whether $\alpha$ can be extended to $x$ as they do not occur in a constraint with $x$. So, it suffices to choose them such that $\alpha$ remains consistent if $\left.\alpha\right|_{\Gamma(x)}$ was consistent. To do this, the algorithm chooses an independent set of size $k-1-\delta$ in $G \backslash \Gamma[S \cup\{x\}]$, which exists and can be obtained greedily due to the lower bound on $|X|$ and because every variable has degree at most $k \cdot \ell$. This terminates the selection of the $k-1$ variables for the scope of $\alpha$. The algorithm then goes over all $d^{k-1}$ partial instantiations with scope $S$. For each such partial instantiation $\alpha$, check in polynomial time whether it is consistent, and if so, whether it can be consistently extended to $x$. If any such check finds that $\alpha$ is consistent, but cannot be consistently extended to $x$, answer No, otherwise answer YES. This part of the algorithm takes time $2^{k \cdot \ell} \cdot d^{k-1} \cdot|N|^{O(1)}$. We conclude that $k$-ConsistenCY, parameterized by $k+d+\ell$, is fixed-parameter tractable.

The algorithm for the Strong $k$-Consistency problem is simpler. After having chosen $x$, there is no need to consider variables that do not occur in a constraint with $x$. To choose $S$, it goes over all subsets of $\Gamma(x)$ of size at most $k-1$, and proceeds as described above.

To solve the Directional $k$-Consistency and Strong Directional $k$-Consistency problems, after having chosen $x$, the algorithm deletes all variables from $N$ that occur after $x$ in the 
ordering $\leq$, and it also removes the constraints whose scope contains at least one of the deleted variables. Then, the algorithm proceeds as above.

Once a local inconsistency in a constraint network is detected, one can add a new (redundant) constraint to the network that excludes this local inconsistency. More specifically, if we detect that a constraint network $N=(X, D, C)$ is not $k$-consistent because some partial instantiation $\alpha$ to a set $S=\left\{x_{1}, \ldots, x_{k-1}\right\}$ of variables cannot be extended to some variable $x$, we add the redundant constraint $\left(\left(x_{1}, \ldots, x_{k-1}\right), D^{k-1} \backslash\left\{\left(\alpha\left(x_{1}\right), \ldots, \alpha\left(x_{k-1}\right)\right)\right\}\right)$ to the network. We repeat this process until we end up with a network $N^{*}$ that is $k$-consistent. One says that $N^{*}$ is obtained from $N$ by enforcing $k$-consistency [2]. Similar notions can be defined for strong/directional $k$-consistency.

It is obvious that the computational task of enforcing $k$-consistency is at least as hard as deciding $k$-consistency. Hence, by Theorems 1 and 3, enforcing (strong/directional) $k$-consistency is co-W[1]hard when parameterized by $k+d$ and co-W[2]-hard when parameterized by $k$.

The fixed-parameter tractability result of Theorem 5 does not directly apply to enforcing, since one can construct instances with small $d$ and $\ell$ that require the addition of a large number of redundant constraints that exceeds any fixed-parameter bound. However, we can obtain fixed-parameter tractability by restricting the enforced network $N^{*}$. Let $\ell^{*}$ denote the maximum number of constraints in which a variable occurs after $k$-consistency is enforced. The proof of Theorem 5 shows that enforcing $k$-consistency is fixed-parameter tractable when parameterized by $k+d+\ell^{*}$.

\section{Conclusion}

In recent years numerous computational problems from various areas of computer science have been identified as fixed-parameter tractable or complete for a parameterized complexity class $\mathrm{W}[i]$ or co-W $[i]$. The list includes fundamental problems from combinatorial optimization, logic, and reasoning (see, e.g., Cesati's compendium [5]). Our results place fundamental problems of constraint satisfaction within this complexity hierarchy.

It is perhaps not surprising that the general local consistency problems are fixed-parameter intractable. The drop in complexity from co-W[2] to co-W[1] when we include the domain size as a parameter shows that domain size is of significance for the complexity of local consistency. Somewhat surprising to us is Theorem 5 which shows that under reasonable assumptions there is still hope for fixed-parameter tractability. This result suggests to look for other less restricted cases for which local consistency checking or even enforcing is fixed-parameter tractable. For instance, it would be interesting to see if Theorem 5 still holds if we replace $\ell$ with the average number of constraints in which a variable occurs.

\section{References}

[1] A. Atserias, A. A. Bulatov, and V. Dalmau. On the power of $k$-consistency. In L. Arge, C. Cachin, T. Jurdzinski, and A. Tarlecki, editors, Automata, Languages and Programming, 34th International Colloquium, ICALP 2007, Wroclaw, Poland, July 9-13, 2007, Proceedings, volume 4596 of Lecture Notes in Computer Science, pages 279-290. Springer Verlag, 2007.

[2] C. Bessiere. Constraint propagation. In F. Rossi, P. van Beek, and T. Walsh, editors, Handbook of Constraint Programming, chapter 3. Elsevier, 2006.

[3] L. Cai, J. Chen, R. G. Downey, and M. R. Fellows. On the parameterized complexity of short computation and factorization. Archive for Mathematical Logic, 36(4-5):321-337, 1997.

[4] M. Cesati. The Turing way to parameterized complexity. J. of Computer and System Sciences, $67: 654-685,2003$

[5] M. Cesati. Compendium of parameterized problems. http://bravo.ce.uniroma2.it/home/cesati/ research/compendium.pdf, Sept. 2006. 
[6] M. Cesati and M. D. Ianni. Computation models for parameterized complexity. Mathematical Logic Quarterly, 43:179-202, 1997.

[7] Y. Chen and J. Flum. The parameterized complexity of maximality and minimality problems. Annals of Pure and Applied Logic, 151(1):22-61, 2008.

[8] M. C. Cooper. An optimal k-consistency algorithm. Artificial Intelligence, 41(1):89-95, 1989.

[9] R. De La Briandais. File searching using variable length keys. In Papers presented at the the March 3-5, 1959, Western Joint Computer Conference, IRE-AIEE-ACM '59 (Western), pages 295-298, New York, NY, USA, 1959. ACM.

[10] R. Dechter. From local to global consistency. Artificial Intelligence, 55(1):87-107, 1992.

[11] R. Dechter. Constraint Processing. Morgan Kaufmann, 2003.

[12] R. Dechter and J. Pearl. Network-based heuristics for constraint-satisfaction problems. Artificial Intelligence, 34(1):1-38, 1987.

[13] R. G. Downey and M. R. Fellows. Fixed-parameter tractability and completeness. In Proceedings of the Twenty-first Manitoba Conference on Numerical Mathematics and Computing (Winnipeg, $M B, 1991)$, volume 87, pages 161-178, 1992.

[14] R. G. Downey and M. R. Fellows. Fixed-parameter tractability and completeness. II. On completeness for $W[1]$. Theoretical Computer Science, 141(1-2):109-131, 1995.

[15] R. G. Downey and M. R. Fellows. Parameterized Complexity. Monographs in Computer Science. Springer Verlag, New York, 1999.

[16] R. G. Downey, M. R. Fellows, and C. McCartin. Parameterized approximation problems. In Parameterized and Exact Computation, Second International Workshop, IWPEC 2006, volume 4169 of Lecture Notes in Computer Science, pages 121-129. Springer Verlag, 2006.

[17] J. Flum and M. Grohe. Parameterized Complexity Theory, volume XIV of Texts in Theoretical Computer Science. An EATCS Series. Springer Verlag, Berlin, 2006.

[18] E. Fredkin. Trie memory. Communications of the ACM, 3:490-499, 1960.

[19] E. C. Freuder. Synthesizing constraint expressions. Communications of the ACM, 21(11):958-966, 1978.

[20] E. C. Freuder. A sufficient condition for backtrack-bounded search. J. of the ACM, 32(4):755-761, 1985.

[21] M. R. Garey and D. R. Johnson. Computers and Intractability. W. H. Freeman and Company, New York, San Francisco, 1979.

[22] C. Komusiewicz, R. Niedermeier, and J. Uhlmann. Deconstructing intractability - a multivariate complexity analysis of interval constrained coloring. J. Discrete Algorithms, 9(1):137-151, 2011.

[23] A. K. Mackworth. Consistency in networks of relations. Artificial Intelligence, 8:99-118, 1977.

[24] U. Montanari. Networks of constraints: fundamental properties and applications to picture processing. Information Sciences, 7:95-132, 1974.

[25] R. Niedermeier. Invitation to Fixed-Parameter Algorithms. Oxford Lecture Series in Mathematics and its Applications. Oxford University Press, Oxford, 2006. 